\title{
Evolución de los modelos sobre el afrontamiento del estrés: hacia el coping positivo
}

\author{
Alberto Ortega Maldonado \\ aortega@uji.es \\ MARISA SALANOVA SORIA \\ salanova@uji.es
}

\section{Resumen}

Introducción: Esta revisión teórica sintetiza la evolución de los principales hallazgos científicos sobre el estrés y las estrategias y habilidades para su afrontamiento (coping). Se presentan de forma paralela tanto los diferentes modelos teóricos utilizados por los investigadores, como la evidencia que se ha ido acumulando mediante la investigación al respecto. Resultados: De la revisión se concluye que la evolución de los estudios en esta materia ha venido demostrando la coocurrencia de emociones negativas y positivas en el manejo de situaciones percibidas como estresantes. Estos resultados han derivado en la elaboración de propuestas que contemplan las emociones positivas como un elemento clave a la hora de afrontar eficazmente situaciones estresantes. En este sentido actualmente se plantea el concepto de coping positivo como aquel en el que se acepta la situación estresante, contemplándola como un reto u oportunidad, posibilitando así la aparición de emociones positivas que permitan escoger la estrategia de afrontamiento más eficaz. Conclusión: Desde una perspectiva aplicada es relevante profundizar en el conocimiento del rol de las emociones positivas en el proceso de afrontamiento al estrés, para diseñar intervenciones eficaces dirigidas al incremento de los niveles de bienestar personal.

Palabras clave: coping, estrategias de afrontamiento, coping positivo, emociones positivas, estrés, bienestar.

\footnotetext{
Abstract

Introduction: This theoretical review summarizes the evolution of the main scientific findings about stress and coping. Different theoretical models used by researchers are presented alongside with the evidence that has been developed during recent years of research. Results: A review of these studies concludes that the evolution of research in this area has been demonstrating the co-occurrence of both negative and positive emotions in stress and coping. These results have led to the development of proposals that consider positive emotions as a key element in selecting and implementing effective strategies for coping. In this regard it emerges nowadays the concept of "positive coping", understood as a kind of coping in which the stressful event is accepted, helping to perceive the situation as a challenge or opportunity, enabling therefore the oc-
} 
currence of positive emotions that allow to choose the most effective coping. Conclusion: From an applied perspective it is very important to broaden the knowledge of the role of positive emotions in the process of stress and coping, in order to design effective interventions aimed at increasing levels of personal wellness.

Keywords: Coping, positive coping, positive emotions, stress, well-being.

\section{Introducción}

Desde una aproximación clásica, se entiende que el estrés se produce cuando en una situación valorada por la persona como significativa, se percibe un desequilibrio entre las demandas de la propia situación y los recursos disponibles para hacerle frente, percibiéndose éstos como insuficientes (Lazarus y Folkman, 1984). Actualmente el estrés y las consecuencias psicológicas y fisiológicas que conlleva, componen uno de los principales problemas y preocupaciones de salud. Por ejemplo, en Europa el estrés constituye el segundo problema de salud que más se denuncia a nivel laboral, generando tanto importantes costes de salud y calidad de vida a las personas, como cuantiosas pérdidas económicas a las administraciones públicas y las empresas privadas (OSHA, 2000; ENWHP, 2009). Es decir, se trata de un aspecto muy relevante para la vida de las personas, presente en nuestro día a día, y determinante para nuestra salud y bienestar.

Pero puesto que sentirse estresado realmente es una característica intrínseca y natural de la vida, el estrés por sí solo no influye directamente en el bienestar general de una persona, sino que como comenta Lazarus (2006), lo realmente trascendente para la felicidad personal es cómo se afrontan las situaciones de estrés. Según el propio Lazarus, si el afrontamiento de una situación estresante es ineficaz, puede derivar en consecuencias perjudiciales para la salud, el bienestar psicológico y el funcionamiento social. Por el contrario, si es eficaz, es probable que se mantenga la situación demandante bajo control, no solo evitando los posibles efectos perniciosos, sino posibilitando incluso crecimiento personal. Por tanto, este afrontamiento eficaz puede conllevar incrementos en los niveles de bienestar a través y a pesar de la situación adversa (Vázquez, Castilla y Hervás, 2007).

Las estrategias de afrontamiento de situaciones estresantes (coping) se definen como aquellos intentos constantes, tanto a nivel cognitivo como comportamental, que realiza una persona para gestionar una situación en la que no se perciben recursos suficientes para su superación (Lazarus y Folkman, 1984; Speirs y Martin, 1999; Zeidner, 1994). De esta forma, resultados de investigaciones al respecto han demostrado que tenemos un amplio repertorio de estrategias para afrontar el estrés, estando su eficacia relacionada con el proceso de ajuste entre las demandas de la situación y la persona, atendiendo a criterios como el bienestar subjetivo, funcionamiento social, o salud fisiológica (French, Caplan y Van Harrison, 1982; Lazarus y Folkman, 1984; Lazarus, 1999). A este respecto, y debido a la necesidad detectada entre otros por Lazarus (2000) y Litman (2006), en los últimos años se han llevado a cabo diversas investigaciones dirigidas a conocer qué estrategias de afrontamiento son más eficaces en la gestión del estrés. En este sentido autores como Folkman (2010) sostienen que el estrés es un fenómeno fundamentalmente contextual, lo que quiere decir que se trata de una transacción o proceso entre la propia persona y el entorno o contexto en el que se encuentra en un momento determinado. Por lo tanto, según esta autora la eficacia de las estrategias de afrontamiento y los resultados asociados a ella dependen tanto de aspectos estables (características de la 
personalidad, valores y creencias personales) como, sobre todo, de elementos inherentes a la propia situación, entre los que destacan las emociones que la persona siente en ese momento (Lazarus, 2000).

Teniendo en cuenta todo lo anterior, y siguiendo a Lazarus (2006), parece razonable la idea de la necesidad de profundizar en el estudio sobre el proceso de afrontamiento al estrés, para conocerlo mejor y determinar cuáles son las estrategias que desempeñamos para gestionar estresores y cuándo y en qué medida son eficaces dichas estrategias. Esta línea de investigación puede ser fundamental para la práctica profesional, pudiendo aportar diseños de intervenciones dirigidas a afrontar eficazmente los momentos estresantes que una persona vive en el día a día, contribuyendo a incrementar sus niveles de bienestar (Eacott y Frydenberg, 2009).

De esta forma Lazarus (2000), en su artículo sobre el estado del arte, apunta que desde sus inicios en los años 40 del pasado siglo hasta la actualidad, la investigación en estrés y coping ha ido incrementándose tanto desde el punto de vista de la cantidad como de la calidad y complejidad de los estudios, lo que ha contribuido a reducir la tradicional brecha entre la investigación y la práctica profesional. Este acrecentamiento del interés y actividad científica relacionada con el estudio del proceso de afrontamiento al estrés, se ha visto además influenciado por el creciente desarrollo de la investigación sobre las emociones positivas que, desde el surgimiento de la psicología positiva (Seligman y Csikszentmihalyi, 2000), también se ha visto incrementado. Así, basándose en la teoría de ampliación y construcción de recursos de las emociones positivas (the broaden-and-build theory of positive emotions) (Fredrickson, 1998, 2001), estudios como el de Tugade, Fredrickson y Barret (2004) han relacionado las emociones positivas y el coping, encontrando que las emociones positivas contribuyen a través de un coping más eficaz al bienestar físico y psicológico de las personas. Más específicamente emociones positivas como el humor, permiten afrontar el estrés de forma más eficaz (Carbelo y Jáuregui, 2006).

Pero, ¿se pueden sentir emociones positivas en una situación estresante? En contra de lo que tradicionalmente se ha postulado tanto desde la cultura popular como desde la psicología científica, las emociones positivas coocurren en situaciones angustiosas y estresantes, teniendo la emoción positiva una función adaptativa (Folkman y Moskowitz, 2000, 2004). De hecho, el afecto positivo ayuda a afrontar el estrés de manera efectiva y a superar sus consecuencias nocivas más rápidamente, predisponiendo a evaluar la situación de estrés como un reto, lo que conlleva un incremento de la esperanza y el optimismo, que a su vez genera y sostiene más afecto positivo (Khosla, 2006). En este sentido, Kim, Suh, Kim y Gopalan (2012) encontraron que los jóvenes inmigrantes asiáticos emplean para afrontar el proceso de aculturación, estrategias de afrontamiento con las que experimentan emociones positivas, lo que contribuye a que mantengan un buen nivel de felicidad y bienestar. Es más, incluso en situaciones de estrés severo como en el caso de cuidadores de enfermos graves (Folkman, 1997) o de desempleados por un tiempo prolongado (Kerr, Dattilo y O'Sullivan, 2012), las emociones positivas coocurren con las negativas, contribuyendo a afrontar más eficazmente la situación y a reducir los niveles de malestar en estas personas.

Por tanto, la inclusión de las emociones positivas en los estudios sobre estrés y coping, puede considerarse como un elemento de ruptura en esta línea de investigación, lo que ha podido influir en el desarrollo de las diferentes propuestas teóricas sobre el afrontamiento. Como aportan Vázquez, Pérez y Sales (2003), los estados positivos proporcionan mejor resistencia ante estresores intensos cuando no solo se perciben las consecuencias negativas de lo sucedido, sino que también se aprecia y valora aquellos elementos positivos de la situación. Por lo que los modelos sobre el proceso de estrés coping han podido ser revisados en consonancia con la evidencia empírica sobre las emociones positivas y las situaciones de estrés. 
Por este motivo, el objetivo de este trabajo es realizar una revisión de las principales propuestas teóricas sobre las estrategias de afrontamiento al estrés, analizar su evolución e indagar sobre la inclusión de emociones positivas en estos modelos, para conocer el rol que se les atribuye en el proceso de estrés y coping.

\section{Método}

El presente estudio consiste en una revisión bibliográfica que pretende analizar y sintetizar el estado de la literatura relacionado con el proceso de percepción y afrontamiento del estrés.

La revisión de bibliografía se llevó a cabo siguiendo secuencialmente dos estrategias de búsqueda. En primera instancia, se realizó búsqueda inicial a través de tres bases de datos: PsycNet, Medline y Pubmed. Los criterios de búsqueda que se emplearon respondieron a diferentes combinaciones de las palabras clave stress, coping, positive coping y positive emotion. La búsqueda de las publicaciones revisadas se limitó a publicaciones que emplean el método de revisión de pares en el proceso de evaluación y aceptación de los trabajos.

Posteriormente, se revisaron las referencias bibliográficas de los artículos seleccionados, las cuales facilitaron el acceso a otros estudios revisados.

Los estudios incluidos en la revisión correspondieron tanto a propuestas teóricas, como revisiones o estudios empíricos relacionados con el proceso de afrontamiento al estrés.

\section{Resultados}

La búsqueda dio como resultado la obtención de tres tipos de aportaciones: artículos empíricos centrados en conocer la relación entre las emociones positivas el estrés y el coping, trabajos teóricos sobre propuestas de modelos, y ensayos sobre el estado de la cuestión.

La totalidad de propuestas se centran en la idea de que existe una amplia gama de repertorios de estrategias de afrontamiento al estrés, que se emplean según las características y habilidades personales, y la situación demandante. Esta idea se basa en los resultados del análisis factorial realizado en los trabajos originarios sobre herramientas de medida de coping. Estos instrumentos coinciden en la base teórica en la que se soportan: modelo transaccional de estrés y afrontamiento (Lazarus, 1966; Folkman y Lazarus, 1980; Lazarus y Folkman, 1984; Folkman 1997; Folkman, 2008, Folkman, 2010). Pese a ello han obtenido resultados dispares en los análisis de factorización, lo que ha influido en que difieran en su propuesta sobre los tipos y la clasificación de las estrategias de afrontamiento. Las tres herramientas de medición de referencia en la mayoría de investigaciones son:

- Wcc: Ways of coping checklist (Folkman y Lazarus, 1980).

- WAYS: Ways of coping questionnaire (Folkman y Lazarus; 1985, 1988)

- COPE Inventory (Carver, Scheier y Weintraub, 1989; Carver, 1997).

Pero la propuesta teórica sobre el estrés y su afrontamiento de Lazarus no constituye tan solo el armazón en el que se basa el diseño de las principales herramientas de medición. La totalidad de los trabajos encontrados en esta revisión (tanto teóricos como empíricos) parten de este modelo de evaluación cognitiva del estrés. Los artículos teóricos relacionados con este modelo muestran que el mismo ha ido sufriendo modificaciones a lo largo de los años. Así inicialmente el modelo no contemplaba la coocurrencia de emociones positivas y negativas 
en el proceso de afrontamiento al estrés. Pero en sucesivas actualizaciones, se han incluido las emociones positivas en el mismo, planteando finalmente que la evaluación de la situación estresante conlleva tanto emociones negativas como positivas. Este hecho ha posibilitado que se amplíen las categorías tradicionales de afrontamiento (centrado en solventar el problema o en disminuir el malestar emocional), para incluir una nueva: el afrontamiento centrado en el significado (véase figura 1).

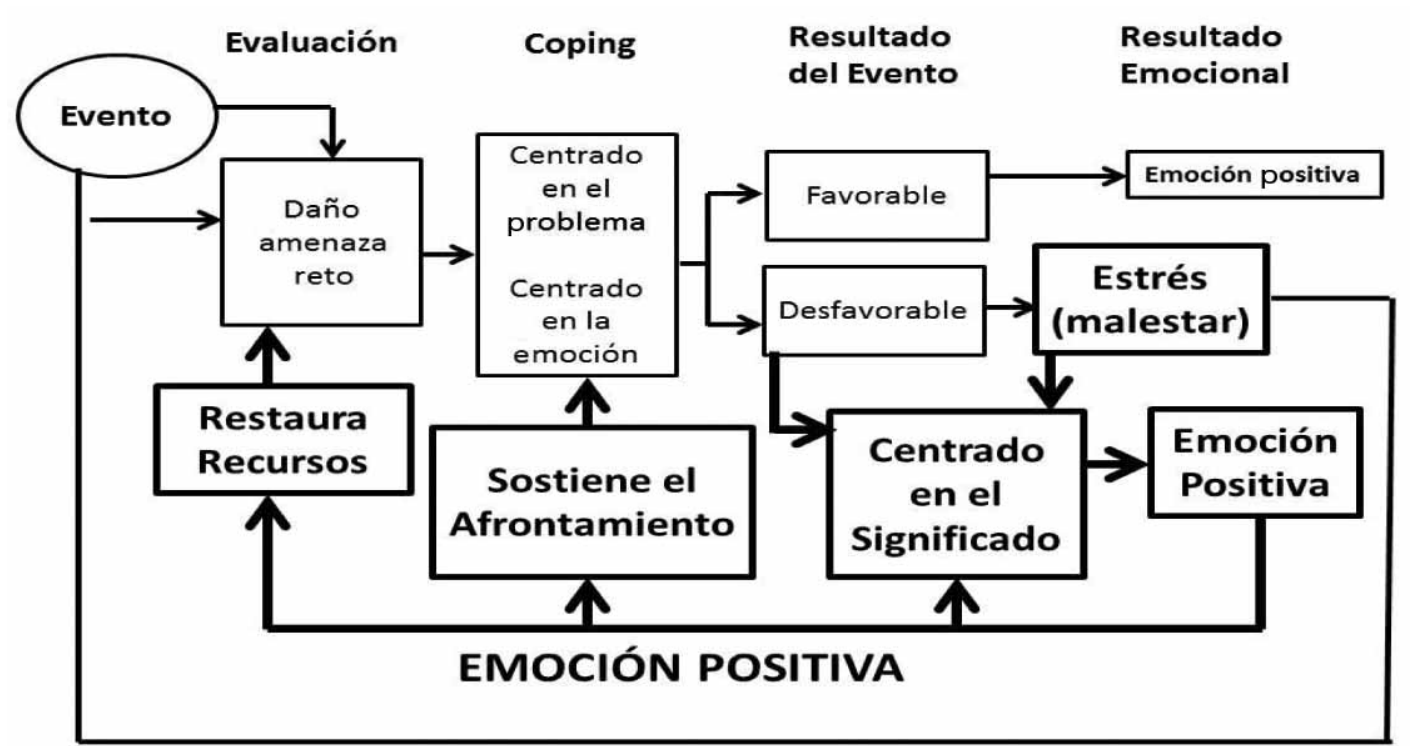

Emoción negativa

Figura 1. Modelo sobre el estrés y coping (Folkman, 2008)

\section{Discusión y conclusiones}

Los resultados de esta revisión acerca de la evolución y desarrollo de los modelos teóricos y estudios empíricos sobre el proceso de estrés y afrontamiento, muestran la inclusión tanto en un tipo de trabajos como en otro, de las emociones positivas como elemento fundamental en dicho proceso. Esta inclusión, derivada de los hallazgos sobre la coocurrencia de emociones negativas y positivas en situaciones percibidas como adversas y demandantes, ha cambiado tajantemente la concepción del proceso de estrés y su afrontamiento. La evidencia empírica apoya la idea plasmada en el modelo base del que parten los trabajos de investigación encontrados: el modelo transaccional de estrés y afrontamiento (Lazarus, 1966; Folkman y Lazarus, 1980; Lazarus y Folkman, 1984; Folkman 1997, 2008, 2010). Los resultados de investigación muestran que las emociones positivas, además de estar presentes en el proceso de afrontamiento al estrés (Folkman y Moskowitz, 2000; Khosla, 2006; Moore y cols., 2014), actúan con un efecto restaurador y potenciador sobre las estrategias de afrontamiento centradas en el problema o en la reducción del malestar, facilitando la eficacia del afrontamiento y los resultados saludables en los que se traduce (Lazarus, Kanner y Folkman, 1980; Folkman, 1997, 2008, 2010). 
Tabla 1

Estrategias de afrontamiento positivo

\begin{tabular}{|c|c|}
\hline Estrategia & Descripción \\
\hline $\begin{array}{l}\text { Reevaluación y reinterpreta- } \\
\text { ción positiva }\end{array}$ & $\begin{array}{l}\text { Encontrar sentido a la situación a través de la interpretación de la } \\
\text { situación en términos de valores y creencias profundamente } \\
\text { arraigadas (Folkman, 1997; Folkman, Moskowitz, Ozer, y Park, } \\
\text { 1997). }\end{array}$ \\
\hline Creencias espirituales & $\begin{array}{l}\text { Buscar significado existencial para conseguir ajuste emocional (Folk- } \\
\text { man, 1997; Folkman, Moskowitz, Ozer, y Park, 1997). }\end{array}$ \\
\hline $\begin{array}{l}\text { Saboreo de los aconte- } \\
\text { cimientos ordinarios positi- } \\
\text { vos }\end{array}$ & $\begin{array}{l}\text { Planear y realizar actividades cotidianas infundiéndole significado } \\
\text { positivo (Folkman y Moskowitz, 2000). }\end{array}$ \\
\hline Aceptación & $\begin{array}{l}\text { Asumir la diferencia y el cambio entre las expectativas generadas } \\
\text { y la situación presente (Folkman, 2008). }\end{array}$ \\
\hline Revisión de las metas & $\begin{array}{l}\text { Adaptar las metas a la nueva situación para mantener la sensa- } \\
\text { ción de control y logro (Folkman, 1997; Folkman, Moskowitz, Ozer, } \\
\text { y Park, 1997). }\end{array}$ \\
\hline $\begin{array}{l}\text { Focalización en los aspectos } \\
\text { positivos }\end{array}$ & $\begin{array}{l}\text { Atender a los aspectos positivos de la situación para permanecer } \\
\text { optimista en cuanto a su solución (Eacott y Frydenberg, 2009). }\end{array}$ \\
\hline $\begin{array}{l}\text { Participación en actividades } \\
\text { de ocio o recreativas }\end{array}$ & $\begin{array}{l}\text { Realizar actividades físicas, relajantes y/o divertidas con perso- } \\
\text { nas cercanas (Eacott y Frydenberg, 2009). }\end{array}$ \\
\hline
\end{tabular}

En este sentido, y a colación de este giro en la concepción del estrés por parte de los teóricos del campo, se propone un nuevo tipo de estrategias de afrontamiento, denominado por Folkman (1997) como coping centrado en el significado, y plasmado en los estudios empíricos revisados y en la psicología positiva aplicada a la práctica profesional como coping positivo (Schwarzer y Knoll, 2003). Esta nueva concepción del afrontamiento de situaciones estresantes ya ha sido puesta en práctica tanto en el ámbito aplicado como en el científico. Así, el coping positivo parece ser un factor relacionado con el bienestar psicológico. Oaksford y cols. (2005) encontraron que el afrontamiento positivo facilita el ajuste psicológico y la adaptación en personas que han sufrido una amputación de alguna extremidad. Kerr y cols., (2012) encontraron mejoras en la salud mental asociadas al empleo de estrategias de coping positivo en personas desempleadas con algún tipo de discapacidad, que padecían estrés crónico. Pero el coping positivo también puede tener consecuencias beneficiosas a nivel fisiológico. Por ejemplo, Sakami y cols. (2003) hallaron que el coping positivo provoca cambios en el sistema inmunológico y efectos favorables para la salud.

Por otra parte, en el ámbito aplicado ya se han desarrollado programas centrados en el desarrollo de habilidades de afrontamiento positivas. Así, Eacott y Frydenmberg (2009) probaron la eficacia de un programa psicoeducativo para desarrollo de habilidades de afrontamiento positivo para jóvenes, tanto para población general como con riesgo de depresión. El programa incluía el entrenamiento en las estrategias de centrarse en la solución del problema, consecución de logros, focalización en lo positivo, búsqueda de actividades divertidas y relajantes, ac- 
tividad física y fomento de las relaciones sociales. Tras la aplicación se obtuvieron resultados tanto en su eficacia (incremento del uso de estrategias de afrontamiento productivas y reducción de las no productivas) como en los efectos sobre la población al que se le aplicó (ajuste persona-situación, bienestar subjetivo y social).

Pero esta nueva tendencia de investigación aún precisa de mucho más profundidad en cuanto al conocimiento generado sobre el coping positivo y sus efectos en el bienestar de las personas. Si bien es cierto que los estudios citados anteriormente evidencian que las emociones positivas generadas por este estilo de afrontamiento incrementan la eficacia a la hora de gestionar el estrés, no existe aún consenso en qué estrategias pueden identificarse con este tipo de coping. Entre las propuestas se encuentran tanto estrategias cognitivas o conductuales centradas en la situación estresante, como otras dirigidas a la generación de emociones positivas, como pueden ser la práctica de la amabilidad, la gratitud el mindfulness, o el desarrollo intencional de fortalezas personales. Por tanto, este nicho de investigación constituye un elemento clave para la ampliación sobre el conocimiento acerca de las estrategias de afrontamiento positivo. Se plantea necesario para un futuro cercano, precisar qué estrategias se pueden clasificar como coping positivo y qué eficacia tiene cada una de ellas en diferentes poblaciones. La transferencia de los resultados de estas investigaciones al ámbito de la práctica profesional se antoja decisiva a la hora de promover en la población un afrontamiento de los problemas diarios orientado a un mayor nivel de bienestar personal.

La propuesta del coping positivo es una buena oportunidad para fomentar bienestar, desarrollo, crecimiento y florecimiento personal en las sociedades. La psicología positiva y los investigadores y profesionales que nos dedicamos a ella contemplamos como se abre un campo de actuación muy interesante y enriquecedor, que puede ser determinante a la hora de contribuir a la felicidad desde ámbitos como la psicología de la educación, la psicología de la salud, o la psicología del trabajo. Depende de nuestro esfuerzo que este barco llegue a buen puerto. Hasta entonces, deberemos de aceptar la situación en la que nos encontramos, reinterpretar la misma y planificar la vía de consecución más acorde a nuestros recursos para llegar hasta nuestra meta.

\section{Referencias bibliográficas}

Carbelo, B. y Jáuregui, E. (2006). Emociones positivas: humor positivo. Papeles del psicólogo, 27, 18-30.

Carver, C. S. (1997). You want to measure coping but your protocol'too long: Consider the brief cope. International Journal of Behavioral Medicine, 4, 92-100.

Carver, C. S., Scheier M. F. y Weintraub J. K. (1989). Assessing coping strategies: a theoretically based approach. Journal of Personal and Social Psychology, 56, 267-83.

Eacott, C. y Frydenberg, E. (2009). Promoting positive coping skills for rural youth: Benefits for at-risk young people. The Australian Journal of Rural Health, 17, 338-345.

ENWHP: Red Europea de Promoción de la Salud en el trabajo (2009). A guide to the business case for mental health. Extraído de http://www.enwhp.org/fileadmin/downloads/8th_Initiative/MentalHealth_Broschuere_bussinescase.pdf.

Folkman, S. (1997). Positive psychological states and coping with severe stress. Social Science and Medicine, 45, 1207-1221.

Folkman, S. (2008). The case for positive emotions in the stress process. Anxiety, Stress, and Coping, 21, 3-14.

Folkman, S. (2010). Stress, coping, and hope. Psychooncology, 19, 901-908. 
Folkman, S. y Lazarus, R. S. (1980). An analysis of coping in a middle-aged community sample. Journal of Health and Social Behavior, 21, 219-239.

Folkman, S. y Lazarus, R. S. (1985). If it changes it must be a process: Study of emotion and coping during three stages of a college examination. Journal of Personality and Social Psychology, 48, 150-170.

Folkman. S. y Lazarus, R. S. (1988). Manual for the ways of coping questionnaire. Palo Alto, CA: Consulting Psychologists Press.

Folkman, S. K. y Moskowitz, J. T. (2000). Positive affect and the other side of coping. American Psychologist, 55, 647-654.

Folkman, S. y Moskowitz, J. T. (2004). Coping: Pitfalls and promise. Annual Review of Psychology, 55, 745-774.

Folkman, S., Moskowitz, J. T., Ozer, E. M. y Park, C. L. (1997). Positive meaningful events and coping in the context of HIV/AIDS. En B. H. Gottlieb (ed.), Coping with chronic stress (pp. 293-314). Nueva York: Plenum Press.

Fredrickson, B. L. (1998). What good are positive emotions? Review of General Psychology, 2, 300-319.

Fredrickson, B. L. (2001). The role of positive emotions in positive psychology: The broadenand-build theory of positive emotions. American Psychologist, 56, 218-226.

French, J. R. P., Caplan, R. B., y Van Harrison, R. (1982). The mechanisms of job stress and strain. Chichester: Wiley.

Kerr, J. L., Dattilo, J., y O'Sullivan, D. (2012). Use of recreation activities as positive coping with chronic stress and mental health outcomes associated with unemployment of people with disabilities. Work: A Journal of Prevention, Assessment and Rehabilitation, 43, 279-292.

Khosla, M. (2006). Positive affect and coping with stress. Journal of the Indian Academy of Applied Psychology, 32, 185-192.

Kim, J., Suh, W., Kim, S., y Gopalan, H. (2012). Coping strategies to manage acculturative stress: Meaningful activity participation, social support, and positive emotion among Korean immigrant adolescents in the USA. International Journal of Qualitative Studies on Health and Well-Being, 7, 1-10.

Lazarus, R. S. (1966). Psychological stress and the coping process. Nueva York: McGrawHill.

Lazarus, R. S. (1999). Hope: an emotion and a vital coping resource against despair. Social Research, 66, 653-678.

Lazarus R. S. (2000). Toward better research on stress and coping. American Psychologist, $55,665-73$.

Lazarus, R. S. (2006). Emotions and interpersonal relationships: toward a person-centered conceptualization of emotions and coping. Journal of personality, 74, 9-46.

Lazarus R. S. y Folkman, S. (1984). Stress, Appraisal, and Coping. Nueva York: Springer.

Lazarus, R. S., Kanner, A. D. y Folkman, S. (1980). Emotions: A cognitive-phenomenological analysis. Theories of Emotion, 1, 189-217.

Litman, J. A. (2006). The COPE inventory: Dimensionality and relationships with approach- and avoidance motives and positive and negative traits. Personality and Individual Differences, 41, 273-284.

Moore, P. J., Chrabaszcz, J. S., Peterson, R. A., Rohrbeck, C. A., Roemer, E. C. y Mercurio, A. E. (2014). Psychological resilience: the impact of affectivity and coping on state anxiety and positive emotions during and after the Washington, DC sniper killings. Anxiety, Stress \& Coping, 27, 138-155. 
Oaksford, K., Frude, N. y Cuddihy, R. (2005). Positive coping and stress-related psychological growth following lower limb amputation. Rehabilitation psychology, 50, 266-277.

OsHA: Agencia Europea para la Seguridad y Salud en el Trabajo (2000). Investigación sobre el estrés relacionado con el trabajo. Extraído de http://osha.europa.eu/en/publications/ reports/203/view.

Sakami, S., Maeda, M., Maruoka, T., Nakata, A., Komaki, G. y Kawamura, N. (2003). Positive coping up-and down-regulates in vitro cytokine productions from $\mathrm{T}$ cells dependent on stress levels. Psychotherapy and psychosomatics, 73, 243-251.

Schwarzer, R. y Knoll, N. (2003). Positive coping: Mastering demands and searching for meaning. En S. J. López y C. R. Snyder (eds.), Positive psychological assessment: A handbook of models and measures., (pp. 393-409). Washington, DC, US: American Psychological Association.

Seligman. M. E. P. y Csikszentmihalyi, M. (2000). Positive psychology: An introduction. American Psychologist, 55, 5-14.

Speirs, T. y Martin, A. J. (1999). Depressed mood amongst adolescents: the roles of perceived control and coping style. Australian Journal of Guidance and Counselling, 9, 55-76.

Tugade, M. M., Fredrickson, B. L. y Feldman Barrett, L. (2004). Psychological resilience and positive emotional granularity: Examining the benefits of positive emotions on coping and health. Journal of personality, 72, 1161-1190.

Vázquez, C., Castilla, C. y Hervás, G. (2008). Reacciones ante el trauma: Resistencia y crecimiento. En E. Fernández-Abascal (eds.), Las emociones positivas. Madrid: Pirámide.

Vázquez, C. y Pérez-Sales, P. (2003). Emociones positivas, trauma y resistencia. Ansiedad y Estrés, 9, 231-254.

Zeidner, M. (1994). Personal and contextual determinants of coping and anxiety in an evaluative situation: A prospective study. Personality and Individual Differences, 16, 899-918. 